\title{
Evidential Reasoning for Multi-Criteria Analysis based on DSmT-AHP
}

\author{
Jean Dezert \\ The French Aerospace Lab. \\ Information Fusion Systems \\ Chemin de la Hunière \\ 91761 Palaiseau Cedex, France. \\ Email: jean.dezert@onera.fr
}

\author{
Jean-Marc Tacnet \\ Cemagref \\ ETGR \\ 2 rue de la Papèterie, B.P. 76 \\ 38402 St. Martin D’Hères Cedex, France. \\ Email: jean-marc.tacnet@cemagref.fr
}

\begin{abstract}
In this paper, we present an extension of the multi-criteria decision making based on the Analytic Hierarchy Process (AHP) which incorporates uncertain knowledge matrices for generating basic belief assignments (bba's). The combination of priority vectors corresponding to bba's related to each (sub)-criterion is performed using the Proportional Conflict Redistribution rule no. 5 proposed in Dezert-Smarandache Theory (DSmT) of plausible and paradoxical reasoning. The method presented here, called DSmT-AHP, is illustrated on very simple examples.
\end{abstract}

Keywords: Analytic Hierarchy Process (AHP), Dezert-Smarandache Theory (DSmT), Evidential Reasoning, Information Fusion, Decision Making, Multi-Criteria Analysis (MCA).

\section{INTRODUCTION}

In many real-life contexts, decisions are based on imperfect information provided by several more or less reliable and conflicting sources. Several recent developments have tried to introduced belief function theory [24] into the AHP framework. A first attempt has been done to consider imprecise evaluations of alternatives using the Dempster-Shafer theory and the Dempster rule [2]. However, the classical fusion rules such as Dempster rule is known to poorly take conflict into account. Another new framework called ER-MCDA has been proposed to mix a multi-criteria decision making method called Analytic Hierarchy Process (AHP) and uncertainty theories including fuzzy sets, possibility and belief function theories [28]. The principle of the $E R-M C D A$ methodology is to use AHP to analyze the decision problem and to replace the aggregation step by two successive fusion processes [29]. Its main objective is to take into account both information imperfection, source reliability and conflict. When doing this, an important problem occurs since the importance of criteria is a different concept than the classical reliability concept developped and used in the belief theory context. This article presents a new development related to the use of fusion in the context of multicriteria decision analysis, focusing on the special case of AHP. First, we present a method called DSmT-AHP which is an adapted version of AHP allowing to consider imprecise and uncertain evaluation of alternatives. Secondly, we describe a new fusion rule that has been developped on the basis of the PCR rule proposed in the context of DSmT Theory to implement this method. This new rule is also used in the context of the ER-MCDA method.

\section{DSMT-AHP APPROACH}

We present briefly the extension of Saaty's AHP method [17] using an aggregation method developed in Dezert-Smarandache Theory (DSmT) framework [25] of evidential reasoning (ER), able to make a difference between importance of criteria, uncertainty related to the evaluations of criteria and reliability of the different sources. This method has been introduced for the first time in [8] and applied for risk prevention of natural hazards in mountains in [29]. Before explaining the principle of DSmT-AHP, it is necessary to recall some basics of DSmT at first to make the paper self-consistent for readers not familiar with this theoretical framework. All basis with many detailed examples can be obtained freely by downloading the three volumes given in [25] from the web.

\section{A. DSmT basics}

Let start with $\Theta=\left\{\theta_{1}, \theta_{2}, \cdots, \theta_{n}\right\}$ be a finite set of $n$ elements assumed to be exhaustive. $\Theta$ corresponds to the frame of discernment of the problem under consideration. In general, we assume that elements of $\Theta$ are non exclusive in order to deal with vague/fuzzy and relative concepts [25], Vol. 2. This is the so-called free-DSm model. In DSmT, there is no need to work on a refined frame consisting in a discrete finite set of exclusive and exhaustive hypotheses (referred as Shafer's model), because DSm rules of combination work for any models of the frame. The hyper-power set $D^{\Theta}$ is defined as the set of all propositions built from elements of $\Theta$ with $\cup$ and $\cap$, see [25], Vol. 1 for examples. A (quantitative) basic belief assignment (bba) expressing the belief committed to the elements of $D^{\Theta}$ by a given source is a mapping $m(\cdot): D^{\Theta} \rightarrow[0,1]$ such that: $m(\emptyset)=0$ and $\sum_{A \in D^{\Theta}} m(A)=1$. Elements $A \in D^{\Theta}$ having $m(A)>0$ are called focal elements of $m($.$) . The credibility$ 
and plausibility functions are defined in almost the same manner as in Dempster-Shafer Theory (DST) [24] except that $2^{\Theta}$ is replaced by $D^{\Theta}$ in their definitions. In DSmT, the Proportional Conflict Redistribution Rule no. 5 (PCR5) is used generally to combine bba's. PCR5 transfers the conflicting mass only to the elements involved in the conflict and proportionally to their individual masses, so that the specificity of the information is entirely preserved in this fusion process. For simplicity, we work in the power set $2^{\Theta}$ since most of readers are usually already familiar with this fusion space. Let's $m_{1}($.$) and m_{2}($.$) be two$ independentbba's, then the PCR5 rule is defined as follows: $m_{P C R 5}(\emptyset)=0$ and $\forall X \in 2^{\Theta} \backslash\{\emptyset\}$

$$
m_{P C R 5}(X)=\sum_{\substack{X_{1}, X_{2} \in 2^{\ominus} \\ X_{1} \cap X_{2}=X}} m_{1}\left(X_{1}\right) m_{2}\left(X_{2}\right)+\sum_{\substack{X_{2} \in 2^{\ominus} \\ X_{2} \cap X=\emptyset}}\left[\frac{m_{1}(X)^{2} m_{2}\left(X_{2}\right)}{m_{1}(X)+m_{2}\left(X_{2}\right)}+\frac{m_{2}(X)^{2} m_{1}\left(X_{2}\right)}{m_{2}(X)+m_{1}\left(X_{2}\right)}\right]
$$

where all denominators in (1) are different from zero. If a denominator is zero, that fraction is discarded. All propositions/sets are in a canonical form. A variant of (1), called PCR6, for combining $s>2$ sources and for working in other fusion spaces (hyper-power sets or super power-sets) is presented in [25]. Additional properties of PCR5 can be found in [7]. Extension of PCR5 for combining qualitative bba's can be found in [25], Vol. $2 \& 3$.

As a simple example, let's consider two bba's $m_{1}($.$) and m_{2}(),. A \cap B=\emptyset$ for the model of $\Theta$, and $m_{1}(A)=0.6$ and $m_{2}(B)=0.3$. With PCR5 the partial conflicting mass $m_{1}(A) m_{2}(B)=0.6 \cdot 0.3=0.18$ is redistributed to $A$ and $B$ only with respect to the following proportions respectively: $x_{A}=0.12$ and $x_{B}=0.06$ because

$$
\frac{x_{A}}{m_{1}(A)}=\frac{x_{B}}{m_{2}(B)}=\frac{m_{1}(A) m_{2}(B)}{m_{1}(A)+m_{2}(B)}=\frac{0.18}{0.9}=0.2
$$

\section{B. DSmT-AHP approach}

DSmT-AHP aimed to perform a similar purpose as AHP [16], [17], SMART [30] or DS/AHP [2], [4], etc. that is to find the preferences rankings of the decision alternatives (DA), or groups of DA. DSmT-AHP approach consists in three steps:

- Step 1: We extend the construction of the matrix for taking into account the partial uncertainty (disjunctions) between possible alternatives. If no comparison is available between elements, then the corresponding elements in the matrix is zero. Each bba related to each (sub-) criterion is the normalized eigenvector associated with the largest eigenvalue of the "uncertain" knowledge matrix (as done in standard AHP approach).

- Step 2: We use the DSmT fusion rules, typically the PCR5 rule, to combine bba's drawn from step 1 to get a final MultiCriteria Decision-Making (MCDM) priority ranking. This fusion step must take into account the different importances (if any) of criteria as it will be explained in the sequel.

- Step 3: Decision-making can be done based either on the maximum of belief (also called credibility), or on the maximum of the plausibility of Decision alternatives (DA), as well as on the maximum of the approximate subjective probability of DA obtained by different probabilistic transformations like the Pignistic, DSmP, or Sudano's transformations [25], Vol. 2.

The MCDM problem deals with several criteria having different importances and the classical fusion rules cannot be applied directly as in step 2. In AHP, the fusion is done from the product of the bba's matrix with the weighting vector of criteria. Such AHP fusion is nothing but a simple componentwise weighted average of bba's and it doesn't actually process efficiently the conflicting information between the sources. It doesn't preserve the neutrality of a full ignorant source in the fusion. To palliate these problems, we recall the new solution for combining sources of different importances proposed in [26]. The reliability is an objective property of a source, whereas the importance of a source is a subjective characteristic expressed by the fusion system designer. The reliability of a source represents its ability to provide the correct assessment/solution of the given problem. It is characterized by a discounting reliability factor, usually denoted $\alpha$ in $[0,1]$, which should be estimated from statistics when available, or by other techniques [12]. The reliability can be context-dependent. By convention, we usually take $\alpha=1$ when the source is fully reliable and $\alpha=0$ if the source is totally unreliable. The reliability of a source is usually taken into account with Shafer's discounting method [24] defined by:

$$
\left\{\begin{array}{l}
m_{\alpha}(X)=\alpha \cdot m(X), \quad \text { for } X \neq \Theta \\
m_{\alpha}(\Theta)=\alpha \cdot m(\Theta)+(1-\alpha)
\end{array}\right.
$$

The importance of a source is not the same as its reliability and we characterize it by an importance factor $\beta$ in $[0,1]$ which represents somehow the weight of importance granted to the source by the fusion system designer. The choice of $\beta$ is usually not related with the reliability of the source and can be chosen to any value in $[0,1]$ by the designer for his/her own reason. By convention, the fusion system designer will take $\beta=1$ when he/she wants to grant the maximal importance of the source in the fusion process, and will take $\beta=0$ if no importance at all is granted to this source in the fusion process. The fusion designer must be able to deal with importance factors in a different way than with reliability factors since they correspond to distinct properties associated with a source of information. The importance of a source is particularly crucial in hierarchical multicriteria decision making problems, specially in the AHP [17], [21]. That's why it is primordial to show how the importance 
can be efficiently managed in evidential reasoning approaches. To take into account the importance of the sources we use the dual of Shafer's discounting approach for reliabilities of sources as originally introduced briefly by Tacnet in [25], Vol.3, Chap. 23 , p. 613. It consists to define the importance discounting with respect to the empty set rather than the total ignorance $\Theta$ (as done with Shafer's discounting). Such new discounting deals easily with sources of different importances and is very simple to use. Mathematically, we define the importance discounting of a source $m($.$) having the importance factor \beta$ in $[0,1]$ by:

$$
\left\{\begin{array}{l}
m_{\beta}(X)=\beta \cdot m(X), \quad \text { for } X \neq \emptyset \\
m_{\beta}(\emptyset)=\beta \cdot m(\emptyset)+(1-\beta)
\end{array}\right.
$$

Here we allow to deal with non-normal bba since $m_{\beta}(\emptyset) \geq 0$ as suggested by Smets in [27]. This new discounting preserves the specificity of the primary information since all focal elements are discounted with same importance factor. Here we use the positive mass of the empty set as an intermediate/preliminary step of the fusion process. Clearly when $\beta=1$ is chosen by the fusion designer, it will mean that the source must take its full importance in the fusion process and so the original bba $m($.$) is kept unchanged. If the fusion designer takes \beta=0$, one will deal with $m_{\beta}(\emptyset)=1$ which is interpreted as a fully non important source. $m(\emptyset)>0$ is not interpreted as the mass committed to some conflicting information (classical interpretation), nor as the mass committed to unknown elements when working with the open-world assumption (Smets interpretation), but only as the mass of the discounted importance of a source in this particular context. Based on this discounting, one adapts PCR5 (or PCR6) rule for $N \geq 2$ discounted bba's $m_{\beta, i}(),. i=1,2, \ldots N$ by considering the following extension, denoted PCR5 ${ }_{\emptyset}$, defined by: $\forall X \in 2^{\Theta}$

$$
m_{P C R 5_{\emptyset}}(X)=\sum_{\substack{X_{1}, X_{2} \in 2^{\Theta} \\ X_{1} \cap X_{2}=X}} m_{1}\left(X_{1}\right) m_{2}\left(X_{2}\right)+\sum_{\substack{X_{2} \in 2^{\Theta} \\ X_{2} \cap X=\emptyset}}\left[\frac{m_{1}(X)^{2} m_{2}\left(X_{2}\right)}{m_{1}(X)+m_{2}\left(X_{2}\right)}+\frac{m_{2}(X)^{2} m_{1}\left(X_{2}\right)}{m_{2}(X)+m_{1}\left(X_{2}\right)}\right]
$$

A similar extension can be done for PCR5 and PCR6 formulas for $N>2$ sources given in [25], Vol. 2. A detailed presentation of this technique with several examples has been done in [26] and thus it is not reported here due to space limitation constraints. The difference between eqs. (1) and (4) is that $m_{P C R 5}(\emptyset)=0$ whereas $m_{P C R 5_{\emptyset}}(\emptyset) \geq 0$. Since we usually work with normal bba's for decision making support, the combined bba will be normalized. In the AHP context, the importance factors correspond to the components of the normalized eigenvector $\mathbf{w}$. It is important to note that such importance discounting method cannot be used in DST when using Dempster-Shafer's rule of combination because this rule is not responding to the discounting of sources towards the empty set (see Theorem 1 in [26] for proof).

We have shown how the reliability and importance of sources can be taken into account in the fusion process separately. The possibility to take them into account jointly is more difficult, because in general the reliability and importance discounting approaches do not commute, but when $\alpha_{i}=\beta_{i}=1$. Indeed, it can be easily verified that $m_{\alpha_{i}, \beta_{i}}(.) \neq m_{\beta_{i}, \alpha_{i}}($.$) whenever$ $\alpha_{i} \neq 1$ and $\beta_{i} \neq 1 . m_{\alpha_{i}, \beta_{i}}($.$) denotes the reliability discounting of m_{i}($.$) by \alpha_{i}$ followed by the importance discounting of $m_{\alpha_{i}}($.$) by \beta_{i}$ which explains the notation $\alpha_{i}, \beta_{i}$ used in index. Similarly, $m_{\beta_{i}, \alpha_{i}}($.$) denotes the importance discounting of$ $m_{i}($.$) by \beta_{i}$ followed by the reliability discounting of $m_{\beta_{i}}($.$) by \alpha_{i}$. In order to deal both with reliabilities and importances factors and because of the non commutativity of these discountings, we have proposed two methods in [26] to proceed the fusion of the sources in a three-steps process as follows:

- Method 1: Step 1: Apply reliability and then importance discountings to get $m_{\alpha_{i}, \beta_{i}}(),. i=1, \ldots, s$ and combine them with $P C R 5_{\emptyset}$ or $P C R 6_{\emptyset}$ and normalize the resulting bba; Step 2: Apply importance and then reliability discountings to get $m_{\beta_{i}, \alpha_{i}}(),. i=1, \ldots, s$ and combine them with $P C R 5_{\emptyset}$ or $P C R 6_{\emptyset}$ and normalize the resulting bba; Step 3 (mixing/averaging): Combine the resulting bba's of Steps 1 and 2 using the arithmetic mean operator ${ }^{1}$.

- Method 2: Another simplest method which could be useful for intermediate traceability in some applications would consist to change Steps $1 \& 2$ by Step 1': Apply reliability discounting only to get $m_{\alpha_{i}}($.$) and combine them with PCR5 or PCR6;$ Step 2': Apply importance discounting only to get $m_{\beta_{i}}($.$) and combine them with P C R 5_{\emptyset}$ or $P C R 6_{\emptyset}$ and normalize the result; Step 3' same as Step 3. Due to space limitation, only Method 1 is briefly illustrated in the following simple example.

\footnotetext{
${ }^{1}$ Other combination rules could be used also like PCR5 or PCR6, etc., but we don't see solid justification to use them again and they require more computations than the simple arithmetic mean.
} 


\section{A simple example}

Let's consider a set of three cars $\Theta=\{A, B, C\}$ with Shafer's model, and only two criteria $\mathrm{C} 1=$ Fuel Economy, C2=Reliability. Let's assume that with respect to each criterion the following "uncertain" preferences matrices are given:

$$
\mathbf{M}(C 1)=\left[\begin{array}{c|ccc} 
& A & B \cup C & \Theta \\
\hline A & 1 & 0 & 1 / 3 \\
B \cup C & 0 & 1 & 2 \\
\Theta & 3 & 1 / 2 & 1
\end{array}\right], \quad \mathbf{M}(C 2)=\left[\begin{array}{c|cccc} 
& A & B & A \cup C & B \cup C \\
\hline A & 1 & 2 & 4 & 3 \\
B & 1 / 2 & 1 & 1 / 2 & 1 / 5 \\
A \cup C & 1 / 4 & 2 & 1 & 0 \\
B \cup C & 1 / 3 & 5 & 0 & 1
\end{array}\right]
$$

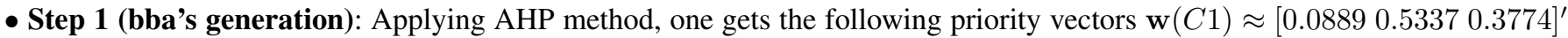
and $\mathbf{w}(C 2) \approx\left[\begin{array}{llll}0.5002 & 0.1208 & 0.1222 & 0.2568\end{array}\right]^{\prime}$ which are identified with the bba's $m_{C 1}($.$) and m_{C 2}($.$) as follows:$ $m_{C 1}(A)=0.0889, m_{C 1}(B \cup C)=0.5337, m_{C 1}(A \cup B \cup C)=0.3774$ and $m_{C 2}(A)=0.5002, m_{C 2}(B)=0.1208$, $m_{C 2}(A \cup C)=0.1222$ and $m_{C 2}(B \cup C)=0.2568$.

- Step 2 (Fusion): When the two criteria have the same full importance in the hierarchy they are fused with one of the classical fusion rules. In [4] Beynon proposed to use Dempster's rule. Here we propose to use the PCR5 fusion rule since it is known to have a better ability to deal efficiently with possibly highly conflicting sources of evidences [25], Vol. 2. With PCR5, one gets:

\begin{tabular}{c|c|c|c} 
Elem. of $2^{\Theta}$ & $m_{C 1}()$. & $m_{C 2}()$. & $m_{P C R 5}()$. \\
\hline$\emptyset$ & 0 & 0 & 0 \\
$A$ & 0.0889 & 0.5002 & 0.3837 \\
$B$ & 0 & 0 & 0.1162 \\
$A \cup B$ & 0 & 0.1208 & 0 \\
$C$ & 0 & 0 & 0.0652 \\
$A \cup C$ & 0 & 0.1222 & 0.0461 \\
$B \cup C$ & 0.5337 & 0.2568 & 0.3887 \\
$A \cup B \cup C$ & 0.3774 & 0 & 0
\end{tabular}

- Step 3 (Decision-making): A final decision based on $m_{P C R 5}($.$) must be taken. Usually, the decision-maker (DM) is$ concerned with a single choice among the elements of $\Theta$. Many decision-making approaches are possible depending on the risk the DM is ready to take. A pessimistic DM will choose the singleton of $\Theta$ giving the maximum of credibility whereas an optimistic DM will choose the element having the maximum of plausibility. A fair attitude consists usually in choosing the maximum of approximate subjective probability of elements of $\Theta$. The result however is very dependent on the probabilistic transformation (Pignistic, DSmP, Sudano's, etc) [25], Vol. 2. We recall ${ }^{2}$ that the credibility Bel(.), the pignistic probability $\operatorname{BetP}($.$) and the plausibility \mathrm{Pl}($.$) functions satisfy for any A \in 2^{\Theta}$ the following inequality:

$$
\operatorname{Bel}(A) \leq \operatorname{BetP}(A) \leq \operatorname{Pl}(A)
$$

where $\operatorname{Bel}(A), \operatorname{Pl}(A)$ and $\operatorname{BetP}(A)$ are defined from any bba $m($.$) by the following formulas:$

$$
\begin{gathered}
\operatorname{Bel}(A)=\sum_{\substack{B \subseteq A \\
B \in 2^{\ominus}}} m(B) \quad \text { and } \quad \operatorname{Pl}(A)=\sum_{\substack{B \cap A \neq \emptyset \\
B \in 2^{\Theta}}} m(B) \\
\operatorname{BetP}(A)=\sum_{Y \in 2^{\ominus}} \frac{|Y \cap A|}{|Y|} m(Y)
\end{gathered}
$$

and where $|Y|$ denotes the cardinality of $Y$. By convention one takes $|\emptyset| /|\emptyset|=1$.

Below are the values of the credibility, the pignistic probability and the plausibility of $A, B$ and $C$ :

\begin{tabular}{c|c|c|c} 
Elem. of $\Theta$ & Bel(.) & BetP(.) & Pl(.) \\
\hline$A$ & 0.3837 & 0.4068 & 0.4298 \\
$B$ & 0.1162 & 0.3105 & 0.5049 \\
$C$ & 0.0652 & 0.2826 & 0.5000
\end{tabular}

The car $A$ will be preferred with the pessimistic (based on max of $\operatorname{Bel}($.$) ) or pignistic attitudes, whereas the car B$ will be preferred if an optimistic attitude is adopted since one has $\mathrm{Pl}(B)>\operatorname{Pl}(C)>\operatorname{Pl}(A)$.

\footnotetext{
${ }^{2}$ In this example, we work with Shafer's model for the frame $\Theta$ so that $D^{\Theta}=2^{\Theta}$.
} 
Now, if we assume that $\mathrm{C} 2$ (the reliability) is three times more important than $\mathrm{C} 1$ (fuel economy) so that the preference matrix between these two criteria is given by:

$$
\mathbf{M}=\left[\begin{array}{ll}
1 / 1 & 1 / 3 \\
3 / 1 & 1 / 1
\end{array}\right] \approx\left[\begin{array}{ll}
1.0000 & 0.3333 \\
3.0000 & 1.0000
\end{array}\right]
$$

Its normalized principal eigenvector is $\mathbf{w}=\left[\begin{array}{lll}0.2500 & 0.7500\end{array}\right]^{\prime}$ which indicates that $\mathrm{C} 2$ is indeed three times more important than $\mathrm{C} 1$ as expressed in the prior DM preferences for ranking criteria. $\mathbf{w}=\left[\begin{array}{ll}w_{1} & w_{2}\end{array}\right]^{\prime}$ can also be obtained directly by solving the algebraic system of equations $w_{2}=3 w_{1}$ and $w_{1}+w_{2}=1$ with $w_{1}, w_{2} \in[0,1]$. If we apply the importance discounting with $\beta_{1}=w_{1}=0.25$ and $\beta_{2}=w_{2}=0.75$, one gets the following discounted bba's

\begin{tabular}{c|c|c} 
Elem. of $2^{\Theta}$ & $m_{\beta_{1}, C 1}()$. & $m_{\beta_{2}, C 2}()$. \\
\hline$\emptyset$ & 0.7500 & 0.2500 \\
$A$ & 0.0222 & 0.3751 \\
$B$ & 0 & 0 \\
$A \cup B$ & 0 & 0.0906 \\
$C$ & 0 & 0 \\
$A \cup C$ & 0 & 0.0917 \\
$B \cup C$ & 0.1334 & 0.1926 \\
$A \cup B \cup C$ & 0.0944 & 0
\end{tabular}

With the PCR5 $5_{\emptyset}$ fusion of the sources $m_{\beta_{1}, C 1}($.$) and m_{\beta_{2}, C 2}($.$) , one gets the results in the table. For decision-making support,$ one prefers to work with normal bba's. Therefore $m_{P C R 5_{\emptyset}}($.$) is normalized by redistributing back m_{P C R 5_{\emptyset}}(\emptyset)$ proportionally to the masses of other focal elements as shown in the right column of the next table.

\begin{tabular}{|c|c|c|}
\hline Elem. of $2^{\Theta}$ & $m_{P C R 5_{\emptyset}}()$. & $m_{P C R 5_{\emptyset}}^{\text {normalized }}()$. \\
\hline$\emptyset$ & 0.6558 & 0 \\
$A$ & 0.1794 & 0.5213 \\
$B$ & 0.0121 & 0.0351 \\
$A \cup B$ & 0.0159 & 0.0461 \\
$C$ & 0.0122 & 0.0355 \\
$A \cup C$ & 0.0161 & 0.0469 \\
$B \cup C$ & 0.1020 & 0.2963 \\
$A \cup B \cup C$ & 0.0065 & 0.0188 \\
\hline
\end{tabular}

If all sources have the same full importances (i.e. all $\beta_{i}=1$ ), then $m_{P C R 5_{\emptyset}}()=.m_{P C R 5}($.$) which is normal because in such$ case $m_{\beta_{i}=1, C i}()=.m_{C i}($.$) . From m_{P C R 5_{\emptyset}}^{\text {normalized }}($.$) one can easily compute the credibility \operatorname{Bel}($.$) , the pignistic probability \operatorname{BetP}($. or the plausibility $\mathrm{Pl}($.$) for each element of \Theta$ for decision-making. In this example one gets:

\begin{tabular}{c|c|c|c} 
Elem. of $\Theta$ & Bel(.) & BetP(.) & $\mathrm{Pl}()$. \\
\hline$A$ & 0.5213 & 0.5741 & 0.6331 \\
$B$ & 0.0351 & 0.2126 & 0.3963 \\
$C$ & 0.0355 & 0.2134 & 0.3974
\end{tabular}

If the classical AHP fusion method (i.e. weighted arithmetic mean) is used directly with bba's $m_{C 1}($.$) and m_{C 2}($.$) , one gets:$

$$
m_{A H P}(.)=\left[\begin{array}{cc}
0 & 0 \\
0.0889 & 0.5002 \\
0 & 0 \\
0 & 0.1208 \\
0 & 0 \\
0 & 0.1222 \\
0.5337 & 0.2568 \\
0.3774 & 0
\end{array}\right] \times\left[\begin{array}{l}
0.25 \\
0.75
\end{array}\right]=\left[\begin{array}{c}
0 \\
0.3974 \\
0 \\
0.0906 \\
0 \\
0.0917 \\
0.3260 \\
0.0944
\end{array}\right]
$$

which would have provided the following result for decision-making

\begin{tabular}{c|c|c|c} 
Elem. of $\Theta$ & Bel(.) & BetP(.) & $\mathrm{Pl}()$. \\
\hline$A$ & 0.3974 & 0.5200 & 0.6741 \\
$B$ & 0 & 0.2398 & 0.5110 \\
$C$ & 0 & 0.2403 & 0.5121
\end{tabular}

In this very simple example, one sees that the importance discounting technique coupled with PCR5-based fusion rule (what we call the DSmT-AHP approach) will suggest, as with classical AHP, to choose the alternative $A$ since the car $A$ has a bigger credibility (as well as a bigger pignistic probability and plausibility) than cars $B$ or $C$. It is however worth to note that the values of $\operatorname{Bel}(),. \operatorname{BetP}($.$) and \operatorname{Pl}($.$) obtained by both methods are slightly different. The difference in results can have a strong$ impact in practice in the final result for example if the costs of vehicles have also to be included in the final decision. Note 
also that the uncertainties $U(X)=\operatorname{Pl}(X)-\operatorname{Bel}(X)$ of all alternatives $X=A, B, C$ have been seriously diminished when using DSmT-AHP with respect to what we obtain with classical AHP as seen in the following table. The uncertainty reduction is a nice expected property specially important for decision-making support.

\begin{tabular}{c|c|c} 
Elem. of $\Theta$ & $U($.$) with AHP$ & $U($.$) with DSmT-AHP$ \\
\hline$A$ & 0.2767 & 0.1118 \\
$B$ & 0.5110 & 0.3612 \\
$C$ & 0.5121 & 0.3619
\end{tabular}

\section{CONCLUSIONS}

In this paper, we have presented a new method for Multi-Criteria Decision-Making (MCDM) and Multi-Criteria Group DecisionMaking (MCGDM) based on the combination of AHP method developed by Saaty and DSmT. The AHP method allows to build bba's from DM preferences of solutions which are established with respect to several criteria. The DSmT allows to aggregate efficiently the (possibly highly conflicting) bba's based on each criterion. This DSmT-AHP method allows to take into account also the different importances of the criteria and/or of the different members of the decision-makers group. The application of this DSmT-AHP approach and specially the new fusion rule has been introduced in an application case for the prevention of natural hazards in mountains and snow avalanches, see [25], Vol.3, Chap. 23, and [29].

\section{REFERENCES}

[1] J. Barzilai, F.A. Lootsma, Power relations and group aggregation in the multiplicative AHP and SMART, J. of MCDA, Vol. 6, pp. 155-165, 1997.

[2] M. Beynon, B. Curry, P.H. Morgan, The Dempster-Shafer theory of evidence: An alternative approach to multicriteria decision modelling, Omega, Vol. 28, No. 1, pp. 37-50, 2000.

[3] M. Beynon, D. Cosker, D. Marshall, An expert system for multi-criteria decision making using Dempster-Shafer theory, Expert Syst. with Appli., Vol. 20, No. 4, pp. 357-367, 2001.

[4] M. Beynon, DS/AHP method: A mathematical analysis, including an understanding of uncertainty, Eur. J. of Oper. Res., Vol. 140, pp. 148-164, 2002.

[5] M. Beynon, Understanding local ignorance and non-specificity within the DS/AHP method of multi-criteria decision making, Eur. J. of Oper. Res., Vol. 163, pp. 403-417, 2005.

[6] M. Beynon, A method of aggregation in DS/AHP for group decision-making with non-equivalent importance of individuals in the group, Comp. \& Oper. Res., No. 32, pp. 1881-1896, 2005.

[7] J. Dezert, F. Smarandache, Non Bayesian conditioning and deconditioning, International Workshop on Belief Functions, Brest, France, April 2010.

[8] J. Dezert, J.-M. Tacnet, M. Batton-Hubert, F. Smarandache, Multi-criteria decision making based on DSmT-AHP, Int. Workshop on Belief Functions, Brest, France, April 2010.

[9] E.H. Forman, S.I. Gass, The analytical hierarchy process: an exposition, Oper. Res., Vol. 49, No. 4 pp. 46-487, 2001.

[10] R.D. Holder, Some Comment on the Analytic Hierarchy Process, J. of the Oper. Res. Soc., Vol. 41, No. 11, pp. 1073-1076, 1990.

[11] F.A. Lootsma, Scale sensitivity in the multiplicative AHP and SMART, J. of MCDA, Vol. 2, pp. 87-110, 1993.

[12] A. Martin, A.-L. Jousselme, C. Osswald, Conflict measure for the discounting operation on belief functions, Proc. of Fusion 2008, Cologne, Germany, July 2008.

[13] C. K. Murphy, Combining belief functions when evidence conflicts, Dec. Sup. Syst., vol. 29, pp. 1-9, 2000.

[14] J. Perez, Some comments on Saaty's AHP, Manag. Sci., Vol. 41, No. 6, pp. 1091-1095, 1995.

[15] J. Perez, J.L. Jimeno, E. Mokotoff, Another Potential Shortcoming of AHP, TOP: An Official J. of the Spanish Soc. of Stats and Oper. Res., Vol. 14, No. 1 , June, 2006

[16] T.L. Saaty, A scaling method for priorities in hierarchical structures, J. of Math. Psych., Vol. 15, PP. 59-62, 1977.

[17] T.L. Saaty, The Analytical Hierarchy Process, McGraw Hill, New York, 1980.

[18] T.L. Saaty, An exposition of the AHP in reply to the paper "Remarks on the Analytic Hierarchy Process”, Manag. Sci., Vol. 36, No. 3, pp. 259-268, 1990.

[19] T.L. Saaty, Response to Holder's Comments on the Analytic Hierarchy Process, J. of the Oper. Res. Soc., Vol. 42, No. 10, pp. 909-914, October 1991.

[20] T.L. Saaty, Decision Making for Leaders: The Analytic Hierarchy Process for Decisions in a Complex World, RWS Publ., Pittsburgh, PA, USA, 1999.

[21] T.L. Saaty, Fundamentals of decision making and priority theory with the analytic hierarchy process, Vol. VI of the AHP series, RWL Publ., Pittsburgh, PA, USA.

[22] T.L. Saaty, Relative Measurement and its Generalization in Decision Making: Why Pairwise Comparisons are Central in Mathematics for the Measurement of Intangible Factors - The Analytic Hierarchy/Network Process, RACSAM, Vol. 102, No. 2, pp. 251-318, 2008.

[23] S. Schenkerman, Inducement of nonexistent order by the analytic hierarchy process, Dec. Sci., Spring 1997.

[24] G. Shafer, A mathematical theory of evidence, Princeton Univ. Press, 1976.

[25] F. Smarandache, J. Dezert, Advances and applications of DSmT for information fusion (Collected works), Vol. 1-3, ARP, 2004-2009. http://www.gallup.unm.edu/ smarandache/DSmT.htm

[26] F. Smarandache, J. Dezert, J.-M. Tacnet, Fusion of sources of evidence with different importances and reliabilities, in Proceedings of Fusion 2010 Int. Conf., Edinburgh, UK, July 26-29th, 2010.

[27] Ph. Smets Ph., The Combination of Evidence in the Transferable Belief Model, IEEE Trans. PAMI 12, pp. 447-458, 1990.

[28] J.M. Tacnet, M. Batton-Hubert and J. Dezert, "Information fusion for natural hazards in mountains" in Advances and applications of DSmT for Information Fusion-Collected works - Volume 3 - Dezert J., Smarandache F. (Eds.) - American Research Press, Rehoboth, USA., pp. 565-659, 2009.

[29] J.-M. Tacnet, M. Batton-Hubert, J. Dezert, A two-step fusion process for multi-criteria decision applied to natural hazards in mountains, Int. Workshop on Belief Functions, Brest, France, April 2010.

[30] D. Von Winterfeldt, W. Edwards Decision analysis and behavioral research, Cambridge Univ. Press, USA, 1986. 